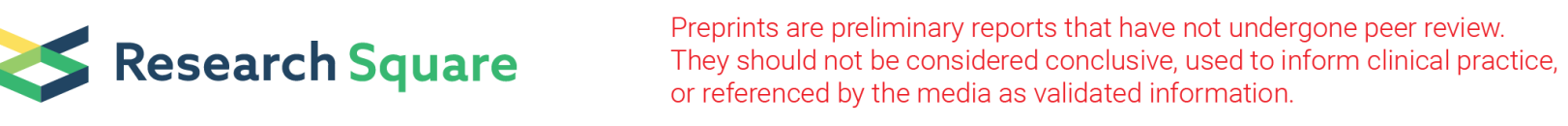

\title{
Percutaneous posterior transiliac plate versus iliosacral screw fixation for posterior fixation of Tile C-type pelvic fractures
}

\section{Chul-Ho Kim}

Chung-Ang University Hospital, Chung- Ang University College of Medicine

Jung Jae Kim

Samsong Seoul Orthopedic Clinic

Ji Wan Kim ( $\sim$ bakpaker@hanmail.net )

University of Ulsan, Asan Medical Center

\section{Research Article}

Keywords: Tile C-type pelvic fractures, iliosacral (IS) screw fixation, percutaneous transiliac plate fixation

Posted Date: May 11th, 2021

DOI: https://doi.org/10.21203/rs.3.rs-497100/v1

License: (c) (i) This work is licensed under a Creative Commons Attribution 4.0 International License.

Read Full License 


\section{Abstract}

Background: This study aimed to compare the clinical outcomes and complications between two minimally invasive surgical techniques: percutaneous transiliac plate fixation and iliosacral (IS) screw fixation for the treatment of Tile C-type pelvic bone fractures.

Materials and Methods: We retrospectively reviewed the data of 77 consecutive patients with Tile $C$ pelvic ring injuries who underwent either percutaneous transiliac plate fixation or IS screw fixation in a single academic center between November 2007 and January 2018. Posterior plating was indicated for those with sacral dysmorphism or bilateral sacral fractures, including spinopelvic dissociation. We recorded patients' demographics, surgery-related data, and postoperative surgical outcomes and compared the incidence of complications and revision surgery rates between the two groups.

Results: Overall, 14 patients were included in the plate group, while 63 were included in the IS screw fixation group. No significant differences were observed in the patients' demographics between the two groups except for a longer interval from injury to surgery ( 13.5 days vs. 5.4 days, $P=0.001$ ). Both groups acquired fracture union in all cases. There was one case of infection requiring surgical debridement in the plating group. Notably, nerve injury $(n=3)$ and implant loosening $(n=5)$ occurred in the IS screw group, but the difference was not significant.

Conclusions: Both percutaneous posterior transiliac plating and IS screw fixation in patients with Tile Ctype pelvic bone fractures showed good results. We recommend IS screw fixation as the primary treatment and propose posterior plating as treatment for sacral dysmorphism and bilateral sacral alar fractures in patients with spinopelvic dissociation.

\section{Introduction}

Tile C-type pelvic bone fractures refer to fractures occurring in the pelvic ring, which are both rotationally and vertically unstable ${ }^{1}$, accompanied by complete disruption of the posterior arch of the pelvic bone. For these fractures, both anterior fixation and posterior fixation are generally required. For posterior fixation, a percutaneous transiliac plate fixation method and iliosacral (IS) screw fixation methods have gained popularity as minimally invasive techniques ${ }^{2}$, but the choice between the two methods remains controversial.

Since the IS screw fixation technique was introduced in the late 1990s, it has become increasingly popular and has shown excellent results over the past few decades ${ }^{3-5}$. The advantages of IS screw fixation are as follows: it can be performed in a supine position, combined anterior fixation can be performed with ease, only a small incision is needed, it is associated with minimal blood loss, and the risk of soft tissue injuries or deep infection is low 6,7 . However, there are also concerns associated with percutaneous IS screw fixation due to the technical demands of the procedure ${ }^{3}$ and the possibility of iatrogenic neurovascular injuries from malpositioned screws ${ }^{8}$. Alternatively, posterior transiliac plate 
fixation allows a more intuitive implant placement and has lower neurovascular damage risk ${ }^{9}$; however, there are concerns regarding other postoperative complications such as infection or pressure sores ${ }^{10,11}$.

Several studies comparing plate fixation and IS screw fixation for the treatment of anterior and posterior pelvic ring fractures have been published. However, studies that provide a direct comparison between the two methods of posterior pelvic ring disruption are limited ${ }^{2,12}$. Therefore, this study aimed to compare the clinical outcomes and complications of these two minimally invasive surgical techniques for the treatment of Tile C-type pelvic bone fractures.

\section{Results}

Among the 203 patients initially screened, 77 were eligible for the analysis (Fig. 1). Of the total study population, 14 patients were included in the plate group and 63 in the IS screw group. Over 31 patients had Tile C1-type (unilateral type) fractures, 36 had Tile C2-type (bilateral type), and 10 had Tile C3-type (bilaterally vertically unstable type). The causes of plate fixation were as follows: dysmorphism $(n=4)$, spinopelvic dissociation with bilateral sacral alar fractures $(n=9)$, and severe AP displacement of more than $10 \mathrm{~mm}(\mathrm{n}=1)$. The mean age of all included patients was 46.5 years (range, $13-84$ years; standard deviation [SD], 16.3). Of the 77 patients, 46 were men and 31 were women. The mean follow-up duration was 36.4 months (range, 1.5-131.9; SD, 32.1); the detailed follow-up durations were 23.9 months in the plate group and 39.4 months in the IS screw group $(p=0.494)$. A comparison of demographic data between the two groups is shown in Table 1. No significant differences were observed in any of the variables between the two groups, except for the time to operation from injury. Patients in the plate group had longer delays than the IS screw group (13.6 vs. 5.4 days, $p=0.001$ ). 
Table 1

Comparison of baseline characteristics between the plate and IS screw groups

\begin{tabular}{|llll|}
\hline & Plate $(\mathbf{n}=\mathbf{1 4})$ & IS screw $(\mathbf{n}=63)$ & P value \\
\hline Age (years) & $48.5 \pm 18.1$ & $46.0 \pm 16.2$ & 0.554 \\
Gender & & & 0.827 \\
Male & $8(57.1 \%)$ & $38(60.3 \%)$ & \\
Female & $6(42.9 \%)$ & $25(39.7 \%)$ & \\
BMI (kg/m $\left.{ }^{2}\right)$ & $25.2($ SD 1.9$)$ & $23.4($ SD 3.5) & 0.110 \\
\hline Injury mechanism (number) & & & 0.425 \\
\hline Traffic accident & $5(35.7 \%)$ & $34(54.0 \%)$ & \\
\hline High altitude falling & $5(35.7 \%)$ & $18(28.6 \%)$ & \\
\hline Crush injury & $4(28.6 \%)$ & $11(17.5 \%)$ & $<0.001$ \\
\hline Time to operation from injury (day) & $13.5($ SD 9.8) & $5.4($ SD 6.6) & \\
\hline Follow-up duration (month) & $23.9($ SD 11.0) & $39.4($ SD 34.9) & 0.494 \\
\hline
\end{tabular}

\section{Perioperative surgery-related data}

Table 2 lists the surgery-related details. In all cases, the anterior pelvic rings were stabilized by external fixation or plating. External fixation was used more frequently than plating in both groups $(78.6 \%$ in the plate group and $87.3 \%$ in the IS screw group); however, the difference was not significant $(p=0.410)$. The mean blood loss volumes were $53.0 \mathrm{~mL}$ in the plate group and $43.5 \mathrm{~mL}$ in the IS screw group, and no significant difference was observed $(p=0.574)$. The mean operation time was longer in the plate group than in the IS screw group (165.3 min vs. $95.9 \mathrm{~min}, p=0.024)$. The length of hospital stay did not differ between the two groups ( 31.6 days vs. 23.2 days, $p=0.282$ ). 
Table 2

Comparison of perioperative surgery-related data and postoperative complications between the two groups

\begin{tabular}{|c|c|c|c|}
\hline & Plate $(n=14)$ & IS screw $(n=63)$ & $P$ value \\
\hline Fixation method of anterior instability ( $\mathrm{n}$ ) & & & 0.410 \\
\hline External fixation & $11(78.6 \%)$ & $55(87.3 \%)$ & \\
\hline Anterior plating & $3(21.4 \%)$ & $8(12.7 \%)$ & \\
\hline Blood loss (mL) & 153.0 (SD 134.2) & 43.5 (SD 21.9) & 0.574 \\
\hline Operation time $(\mathrm{min})$ & 165.3 (SD 89.4) & 95.9 (SD 37.7) & 0.024 \\
\hline Hospital stay (day) & 31.6 (SD 26.9) & 23.2 (SD 18.2) & 0.282 \\
\hline \multicolumn{4}{|l|}{ Postoperative complications (n) } \\
\hline Nerve injury & $0(0 \%)$ & $3(4.8 \%)$ & $>0.999$ \\
\hline Vessel injury & $0(0 \%)$ & $0(0 \%)$ & $>0.999$ \\
\hline Loosening & $0(0 \%)$ & $5(7.9 \%)$ & 0.578 \\
\hline Infection & $1(7.1 \%)$ & $1(1.6 \%)$ & 0.333 \\
\hline Delayed union & $1(7.1 \%)$ & $2(3.2 \%)$ & 0.457 \\
\hline Total & $2(14.3 \%)$ & $11(17.5 \%)$ & $>0.999$ \\
\hline Revision (n) & $1(7.1 \%)$ & $4(6.3 \%)$ & $>0.999$ \\
\hline
\end{tabular}

\section{Postoperative complications and revision rate}

In both groups, fracture union was achieved in all the patients. Three patients experienced delayed union, one from the plate fixation group $(7.1 \%)$ and two from the IS screw fixation group (3.2\%). In the IS screw group, 3 patients (4.8\%) developed postoperative nerve injury, which was not observed in the plate group, although no significant differences were noted between the two groups $(p>0.999)$. Three patients in the plate group complained of new-onset pain on the L5 or S1 dermatome after IS screw fixation, but no motor weakness was observed. Revision surgery for a screw change was performed in only one patient because of a screw breach to the sacral foramen, and the other two patients did not undergo screw change after confirming the absence of screw malplacement on postoperative $\mathrm{CT}$. None of the groups experienced vessel injury. Five patients $(7.9 \%)$ in the IS screw group experienced implant loosening, but none in the plate group $(p=0.578)$. Three of the five patients required revision surgery, two patients required additional posterior plate fixation (Fig. 2), and one required additional IS (S2) screw fixation; in two patients, no other interventions were performed as fracture union was achieved, and no additional interventions were required. One patient in each group developed surgical site infection. In the plate 
group, the infection was deep and was successfully treated with surgical debridement and antibiotics, while one patient with a superficial infection in the IS screw fixation group recovered after receiving intravenous antibiotics. The overall complication rates-which included rates of delayed union, nerve injury related to posterior fixation, vessel injury, implant loosening, and surgical site infections-were $14.3 \%$ in the plate fixation group and $17.5 \%$ in the IS screw fixation group ( $p>0.999)$. One patient $(7.1 \%)$ in the plate fixation group and four (6.3\%) in the IS screw group required revision surgery. No significant differences were found between the two groups. Additional details about perioperative surgery-related data and postoperative complications between the two groups are shown in Table 2.

\section{Discussion}

Our investigation showed that the two fixation methods, percutaneous plate fixation and percutaneous IS screw fixation, were both effective treatment modalities for Tile C-type pelvic bone fractures. No differences were noted in terms of perioperative and postoperative complications, except for longer operation times in the plate group. Both methods demonstrated reliable outcomes, which are supported by previous studies $3,13,14$. IS screw fixation was implemented in patients who required posterior fixation for Tile C-type pelvic ring injury. It is a simple and minimally invasive procedure, but it has limitations as it is difficult to perform in patients with sacral dysmorphism ${ }^{15}$. In addition, this approach has been associated with fixation failure in patients with vertical sacral fractures ${ }^{16}$. Sacral dysmorphism was found in $7-41 \%{ }^{17-19}$ of patients in previous studies, compared with $5.2 \%$ (4 patients) in our study. Although several techniques have been used to insert IS screws within a narrow safe zone, it is technically demanding, and there is potential for screw misplacement. Modified in-out-in corridors can enlarge safe zones, but shortening the length is inevitable, which results in weakening of the fixation power ${ }^{15}$. As another option, the $\mathrm{S} 3$ segment of a dysmorphic sacra can serve as an additional site for screw insertion ${ }^{20}$. Therefore, we chose the plate option in these cases and achieved successful outcomes. Spinopelvic dissociation is another indication for posterior plating. Vertical displacement of the sacral alar was significantly related to IS screw failure ${ }^{16}$, and insertion of a single IS screw would be more risky in patients with bilateral displaced sacral alar fractures. Our study demonstrated three patients with spinopelvic dissociation who achieved good clinical outcomes.

The current study showed no significant difference between the two groups; however, all implant loosening occurred in the IS screw fixation group. A cadaver-biomechanical study revealed that posterior trans-iliosacral plate with additional IS screw was $9 \%$ stronger at $2.5-\mathrm{mm}$ displacement and $6 \%$ increased strength at 5-mm cross-headed displacement compared with a single IS screw ${ }^{21}$. Another biomechanical study using a hard plastic pelvic bone model reported a larger displacement with the IS screw fixation technique than with the posterior tension band plating ${ }^{22}$. Clinical studies have also demonstrated a higher failure rate of IS screw fixation than the plate technique ${ }^{12,23,24}$. As this study showed, we recommend posterior plating in patients with vertically displaced bilateral sacral fractures or in those requiring more rigid fixation, particularly individuals with vertically displaced sacral fractures. 
IS screw fixation carries a higher risk of iatrogenic nerve damage than plate fixation. Neurological complications were reported in up to $8 \%$ of patients who underwent IS screw fixation ${ }^{4,25}$. The current study found no significant differences in nerve injury rates between the two methods; however, three patients experienced nerve injuries in the IS screw group. Two previous studies compared the iatrogenic nerve damage rates between the two methods. Li et al. ${ }^{12}$ compared 13 patients who underwent plate fixation and 7 who had IS screw fixation. In this study, the rate of nerve and vessel injuries in the IS screw fixation group was twofold higher than that in the plate fixation group. Chen et al. ${ }^{2}$ reported 2 patients who had nerve injury among the 29 patients from the IS screw fixation group, while none experienced such injury in the percutaneous posterior plate fixation group with a sample size of 29 patients. However, neither of the studies found any significant differences between the two groups, perhaps due to the small sample size. Our study had a larger sample size; however, the results seemed comparable to those of previous studies. In this study, the incidence of iatrogenic nerve injuries was $4.8 \%$. More caution should be applied, and careful examination of fluoroscopic images must be performed ${ }^{26}$. The use of navigation techniques for guidewire placement and intraoperative 3D-image control of the guidewire position are helpful in reducing this complication 27,28 .

There is a consensus that longer operation times are disadvantageous because they lead to higher rates of postoperative infection ${ }^{29}$. This is relevant to our findings regarding operation times in the two treatment groups. For posterior plate fixation, a position change (to prone position) was needed, and at least six screws were fixed, which resulted in longer operation times. Another important finding was that patients developed infections, including back wounds. The rate of wound infection was $7.1 \%$ in the plate group, while it was only $1.6 \%$ in the IS screw group. Fortunately, the patients in the plate group who developed infections only required surgical debridement. Posterior plate fixation requires posterior soft tissue dissection in thin layers, which can put a patient at risk of developing infection. We attempted to place the plate below the PSIS level to minimize the irritation caused by the plate, and a small skin incision was made to carefully dissect the soft tissue and perform tunneling.

A number of studies have investigated the surgical outcomes of posterior plating using a 4.5-mm plate; however, a 3.5-mm reconstruction plate could minimize soft tissue irritation. Previous studies have discussed the occurrence of wound infections following posterior plate fixation, with reported rates of $10.5-30 \% 3,30,31$. The study analyzed the group that underwent insertion of a $4.5-\mathrm{mm}$ plate above the PSIS, which required a longer incision compared with the other group and had a $17.2 \%$ (5 of 29) infection rate with all patients who developed infections requiring surgical debridement ${ }^{30,31}$; meanwhile, one study that involved the insertion of a 3.5-mm plate below the PSIS showed a $4.8 \%$ deep infection rate ${ }^{3}$. A 3.5$\mathrm{mm}$ reconstructed plate was placed below the PSIS and required a small incision, which showed satisfactory results without mechanical failures. In the current study, although we tried to prevent the occurrence of wound complications, one patient with multiple severe traumatic injuries developed an infection due to prolonged bed rest. However, only one patient had postoperative infection (7.1\%), which is a relatively lower rate compared with that reported in previous studies that used a $4.5-\mathrm{mm}$ plate for posterior plate fixation. Therefore, we recommend a $3.5-\mathrm{mm}$ reconstruction plate placed below the PSIS 
to minimize irritation and wound complications. Surgeons should pay close attention to the risk of surgical wounds in patients undergoing posterior transiliac plating.

This study has several limitations. First, the sample size was relatively small; however, to the best of our knowledge, this study has the largest number of study participants to date in order to compare posterior transiliac plate fixation and IS screw fixation. Second, there was an imbalance in the number of patients in the two groups; the plate fixation group had a smaller number of patients than the IS screw group. Third, we could not compare the patient-reported outcome measures (PROM) because of the retrospective nature of the study, and its study population mainly consisted of severely injured patients. Further studies with larger sample sizes and more detailed outcome variables, such as PROM, or well-structured synthetic analysis are needed to draw more definitive conclusions on this topic.

In summary, we recommend IS screw fixation as the primary treatment for posterior pelvic ring fixation in patients with Tile C-type pelvic fractures. Surgeons should consider the risk of screw loosening and iatrogenic nerve injuries. Based on the results of this study, we recommend percutaneous posterior transiliac plate fixation for patients with 1) sacral dysmorphism and 2) bilateral sacral alar fractures (spino-pelvic dissociation) that require a more rigid fixation, including revision.

\section{Methods}

This study was approved by the Institutional Review Board of Asan Medical Center and waiver was received for the need to provide written informed consent. Data collection was performed in accordance with relevant guidelines and regulations of the committee. Data cannot be shared publicly because it contains potentially identifying information of each patient. Data are available from the Asan Medical Center Institutional Data Access / Ethics Committee (contact via Asan Medical Center Institutional Review Board, Convergence Innovation Bldg. 88, Olympic-ro 43-gil, Songpa-gu, Seoul, Republic of Korea. Website link, http://eirb.amc.seoul.kr/; E-mail, irb@amc.seoul.kr; Phone, +82-2-3010-7165).

\section{Patient selection}

We performed a retrospective review of the medical records of all consecutive patients who had undergone percutaneous plate or IS screw fixation for posterior pelvic ring fractures between November 2007 and January 2018 in a university hospital. Of these patients, we included those who 1) had undergone percutaneous transiliac plate fixation or IS screw fixation for a posterior pelvic ring fracture and 2) those who experienced a Tile C-type pelvic ring fracture. We excluded patients who 1) were diagnosed with pelvic insufficiency fractures, 2) had not undergone anterior fixation; these patients were excluded since they had relatively stable injuries, and 3) with displaced sacral U-type fractures; these fractures usually required triangular fixation, which was not sufficiently stabilized with posterior plating or IS screws ${ }^{32}$.

Selection of fixation method, surgical techniques, and postoperative rehabilitations 
All procedures were performed by two experienced faculty surgeons specializing in pelvic trauma surgery. For patients in whom acceptable fracture reduction could be achieved for posterior pelvic ring injuries, either a percutaneous plate fixation or IS screw fixation technique was employed. For patients with sacral dysmorphism, spinopelvic dissociation with bilateral sacral alar fractures, or a high risk of nerve injury due to severe anteroposterior (AP) displacement of $>10 \mathrm{~mm}$, percutaneous plating was preferred over IS screw fixation. Although we applied an individual postoperative rehabilitation protocol, weight-bearing was not permitted until postoperative 4 weeks, and patients were ambulated with non-weight-bearing or used a wheelchair. After confirming the radiologic stability, the patients were allowed tolerable ambulation with gradually increasing weight-bearing.

\section{(1) Percutaneous posterior transiliac plate fixation}

With the patient in the prone position, 3-cm dual vertical incisions were made lateral to the posterior superior iliac spine (PSIS). After the PSIS was exposed, the superficial fascia was incised along the direction of its fibers and retracted. The gluteal muscles were stripped away from the outer plate of the ilium, and pre-bent 3.5-mm reconstruction plates were inserted just below the posterosuperior iliac spine to one side following resection of the S3 spinous process under fluoroscopy. The plate was inserted into the opposite side through the subcutaneous tunnel and placed on the dorsal side of both ilia. Finally, the screws were positioned to penetrate the bony cortex on both sides (Fig. 3$)^{22,33,34}$.

\section{(2) IS screw fixation}

The patients were placed in a supine position on a transparent surgical table. A pad was placed under the lumbar region, and a small incision $(1 \mathrm{~cm})$ was made after identifying the safe zone on the lateral side of the pelvis. A guidewire was inserted through the safe zone and confirmed with $\mathrm{C}$-arm inlet and outlet views ${ }^{4,8}$. After confirming that the cannula was inserted through the incision as far as the ilium, the cannula was positioned parallel to the upper portion of the $\mathrm{S} 1$ vertebra along the superior margin under Carm guidance. The guidewire was inserted from the ilium through the sacrum cross the middle of sacral

body carefully so that it did not breach the sacral foramina, anterior and posterior to the cortex of the sacral body. Following Kirschner wire insertion, cannulated drill holes were made and 7.0- or 7.3-mm cannulated screws were introduced. If the fracture was considered highly unstable, the screws were inserted in the $\mathrm{S} 2$ vertebra in the same manner.

\section{Data collection and statistical analysis}

From the medical records, we extracted the demographic data of both groups, including age, sex, body mass index, injury mechanism, time to surgery following injury, and follow-up duration. The perioperative surgery-related outcomes were assessed: fixation method of the anterior pelvic ring, volume of blood loss, operation time, and length of hospital stay. We reviewed the postoperative outcomes, including union rate, incidence of delayed union, incidence of iatrogenic nerve injury of the L5 or S1 nerve after posterior fixation, rates of vessel injury, incidence of implant loosening, postoperative infection rates, and need for revision surgery. 
To compare variables between the two groups, the Mann-Whitney $U$ tests were used for continuous variables, while the chi-square test or Fisher's exact test was used to evaluate categorical variables, after verifying the assumption that the data follows a normal distribution. As all continuous variables were not normally distributed, the Mann-Whitney $\mathrm{U}$ tests were used to evaluate these variables in the current study. Moreover, the chi-square test was used to compare the two fixation methods of anterior instability, while the Fisher's exact test was used to evaluate other categorical variables after verifying the normality of its distribution. All statistical analyses were performed using PASW Statistics version 18.0 (IBM Corp., Armonk, NY, USA). A p-value of $<0.05$ was considered significant.

\section{Declarations}

\section{Acknowledgements}

None

\section{Author contributions}

C-H.K. data collection, interpretation, performed manuscript preparation, revised final manuscript. JJ.K. data collection, validation. JW.K. conceived the study ideas, data interpretation, manuscript preparation and revision.

\section{Conflict of Interest}

The authors declare that they have no conflict of interest.

\section{Funding}

Ji Wan Kim declares that this study was supported by the Technology Innovation Program (20000397, Development of Two Track Customized 3D Printing Implant Manufacturing and Commercializing Techniques for Complex Bone Fractures) funded by the Ministry of Trade, Industry \& Energy (MOTIE, Korea).

\section{References}

$1 \quad$ Tile, M. Pelvic ring fractures: should they be fixed? J Bone Joint Surg Br 70, 1-12 (1988).

2 Chen, H. W. et al. Treatment of unstable posterior pelvic ring fracture with percutaneous reconstruction plate and percutaneous sacroiliac screws: a comparative study. J Orthop Sci 17, 580-587, doi:10.1007/s00776-012-0257-1 (2012).

3 Ayoub, M. A., Gad, H. M. \& Seleem, O. A. Standalone percutaneous transiliac plating of vertically unstable sacral fractures: outcomes, complications, and recommendations. European Spine Journal 25, 1153-1162, doi:10.1007/s00586-015-3976-0 (2016). 
4 Routt, M. L., Jr., Kregor, P. J., Simonian, P. T. \& Mayo, K. A. Early results of percutaneous iliosacral screws placed with the patient in the supine position. J Orthop Trauma 9, 207-214, doi:10.1097/00005131-199506000-00005 (1995).

5 Routt, M. L., Jr., Nork, S. E. \& Mills, W. J. Percutaneous fixation of pelvic ring disruptions. Clin Orthop Relat Res, 15-29, doi:10.1097/00003086-200006000-00004 (2000).

6 Ruatti, S. et al. Technique for reduction and percutaneous fixation of U- and H-shaped sacral fractures. Orthop Traumatol Surg Res 99, 625-629, doi:10.1016/j.otsr.2013.03.025 (2013).

7 Shuler, T. E., Boone, D. C., Gruen, G. S. \& Peitzman, A. B. Percutaneous iliosacral screw fixation: early treatment for unstable posterior pelvic ring disruptions. J Trauma 38, 453-458, doi:10.1097/00005373-199503000-00031 (1995).

8 Giannoudis, P. V., Tzioupis, C. C., Pape, H. C. \& Roberts, C. S. Percutaneous fixation of the pelvic ring: an update. J Bone Joint Surg Br 89, 145-154, doi:10.1302/0301-620X.89B2.18551 (2007).

9 Krappinger, D. et al. Minimally invasive transiliac plate osteosynthesis for type C injuries of the pelvic ring: A clinical and radiological follow-up. Journal of Orthopaedic Trauma 21, 595-602, doi:10.1097/BOT.0b013e318158abcf (2007).

10 Robles, L. A. Transverse sacral fractures. Spine J 9, 60-69, doi:10.1016/j.spinee.2007.08.006 (2009).

11 Yi, C. \& Hak, D. J. Traumatic spinopelvic dissociation or U-shaped sacral fracture: a review of the literature. Injury 43, 402-408, doi:10.1016/j.injury.2010.12.011 (2012).

$12 \mathrm{Li}, \mathrm{S}$. et al. Effects of minimally invasive plate-screw internal fixation in the treatment of posterior pelvic ring fracture. Exp Ther Med 16, 4150-4154, doi:10.3892/etm.2018.6670 (2018).

13 Beaule, P. E., Antoniades, J. \& Matta, J. M. Trans-sacral fixation for failed posterior fixation of the pelvic ring. Arch Orthop Trauma Surg 126, 49-52, doi:10.1007/s00402-005-0069-2 (2006).

14 Lindsay, A., Tornetta, P., 3rd, Diwan, A. \& Templeman, D. Is Closed Reduction and Percutaneous Fixation of Unstable Posterior Ring Injuries as Accurate as Open Reduction and Internal Fixation? J Orthop Trauma 30, 29-33, doi:10.1097/BOT.0000000000000418 (2016).

15 Wendt, H. et al. Recommendations for iliosacral screw placement in dysmorphic sacrum based on modified in-out-in corridors. J Orthop Res 37, 689-696, doi:10.1002/jor.24199 (2019).

16 Griffın, D. R., Starr, A. J., Reinert, C. M., Jones, A. L. \& Whitlock, S. Vertically unstable pelvic fractures fixed with percutaneous iliosacral screws: does posterior injury pattern predict fixation failure? $J$ Orthop Trauma 17, 399-405, doi:10.1097/00005131-200307000-00001 (2003). 
17 Gras, F. et al. Transsacral Osseous Corridor Anatomy Is More Amenable To Screw Insertion In Males: A Biomorphometric Analysis of 280 Pelves. Clin Orthop Relat Res 474, 2304-2311, doi:10.1007/s11999-016-4954-5 (2016).

18 Kaiser, S. P., Gardner, M. J., Liu, J., Routt, M. L., Jr. \& Morshed, S. Anatomic Determinants of Sacral Dysmorphism and Implications for Safe lliosacral Screw Placement. J Bone Joint Surg Am 96, e120, doi:10.2106/JBJS.M.00895 (2014).

19 Kim, J. J., Jung, C. Y., Oh, H. K., Yang, B. S. \& Chang, J. S. Upper Sacral Morphology Related to Iliosacral Screw Fixation in Korean. J Korean Fract Soc 20, 115-122, doi:10.12671/jkfs.2007.20.2.115 (2007).

20 Hwang, J. S. et al. Safe Zone Quantification of the Third Sacral Segment in Normal and Dysmorphic Sacra. J Orthop Trauma 32, 178-182, doi:10.1097/bot.0000000000001100 (2018).

21 Padalkar, P. et al. Trans-iliosacral plating for vertically unstable fractures of sacral spine associated with spinopelvic dissociation: A cadaveric study. Indian J Orthop 46, 274-278, doi:10.4103/0019-5413.96376 (2012).

22 Yinger, K., Scalise, J., Olson, S. A., Bay, B. K. \& Finkemeier, C. G. Biomechanical comparison of posterior pelvic ring fixation. J Orthop Trauma 17, 481-487, doi:10.1097/00005131-200308000-00002 (2003).

23 Iotov, A., Takov, E., Iranov, V. \& Kastelov, R. Posterior internal fixation of unstable pelvic fractures. Ortopediya i Travmatologiya 34, 108-112 (1998).

24 Liuzza, F. et al. Comparison between posterior sacral plate stabilization versus minimally invasive transiliac-transsacral lag-screw fixation in fractures of sacrum: a single-centre experience. International orthopaedics 43, 177-185, doi:10.1007/s00264-018-4144-z (2019).

25 Schweitzer, D., Zylberberg, A., Cordova, M. \& Gonzalez, J. Closed reduction and iliosacral percutaneous fixation of unstable pelvic ring fractures. Injury 39, 869-874, doi:10.1016/j.injury.2008.03.024 (2008).

26 Kim, J. W. et al. Fluoroscopic Views for a More Accurate Placement of Iliosacral Screws: An Experimental Study. J Orthop Trauma 30, 34-40, doi:10.1097/BOT.0000000000000426 (2016).

27 Matityahu, A. et al. Three-dimensional navigation is more accurate than two-dimensional navigation or conventional fluoroscopy for percutaneous sacroiliac screw fixation in the dysmorphic sacrum: a randomized multicenter study. J Orthop Trauma 28, 707-710, doi:10.1097/bot.0000000000000092 (2014).

28 Moon, S. W. \& Kim, J. W. Usefulness of intraoperative three-dimensional imaging in fracture surgery: a prospective study. J Orthop Sci 19, 125-131, doi:10.1007/s00776-013-0475-1 (2014). 
29 Cheng, H. et al. Prolonged Operative Duration Increases Risk of Surgical Site Infections: A Systematic Review. Surg Infect (Larchmt) 18, 722-735, doi:10.1089/sur.2017.089 (2017).

30 Boudissa, M., Saad, M., Kerschbaumer, G., Ruatti, S. \& Tonetti, J. Posterior transiliac plating in vertically unstable sacral fracture. Orthop Traumatol Surg Res 106, 85-88, doi:10.1016/j.otsr.2019.06.014 (2020).

31 Suzuki, T. et al. Outcome and complications of posterior transiliac plating for vertically unstable sacral fractures. Injury 40, 405-409, doi:10.1016/j.injury.2008.06.039 (2009).

32 Vaccaro, A. R. et al. Description and Reliability of the AOSpine Sacral Classification System. J Bone Joint Surg Am 102, 1454-1463, doi:10.2106/JBJS.19.01153 (2020).

33 Albert, M. J., Miller, M. E., MacNaughton, M. \& Hutton, W. C. Posterior pelvic fixation using a transiliac 4.5-mm reconstruction plate: a clinical and biomechanical study. J Orthop Trauma 7, 226-232, doi:10.1097/00005131-199306000-00005 (1993).

34 Kobbe, P., Hockertz, I., Sellei, R. M., Reilmann, H. \& Hockertz, T. Minimally invasive stabilisation of posterior pelvic-ring instabilities with a transiliac locked compression plate. International orthopaedics 36 , 159-164, doi:10.1007/s00264-011-1279-6 (2012).

\section{Figures}




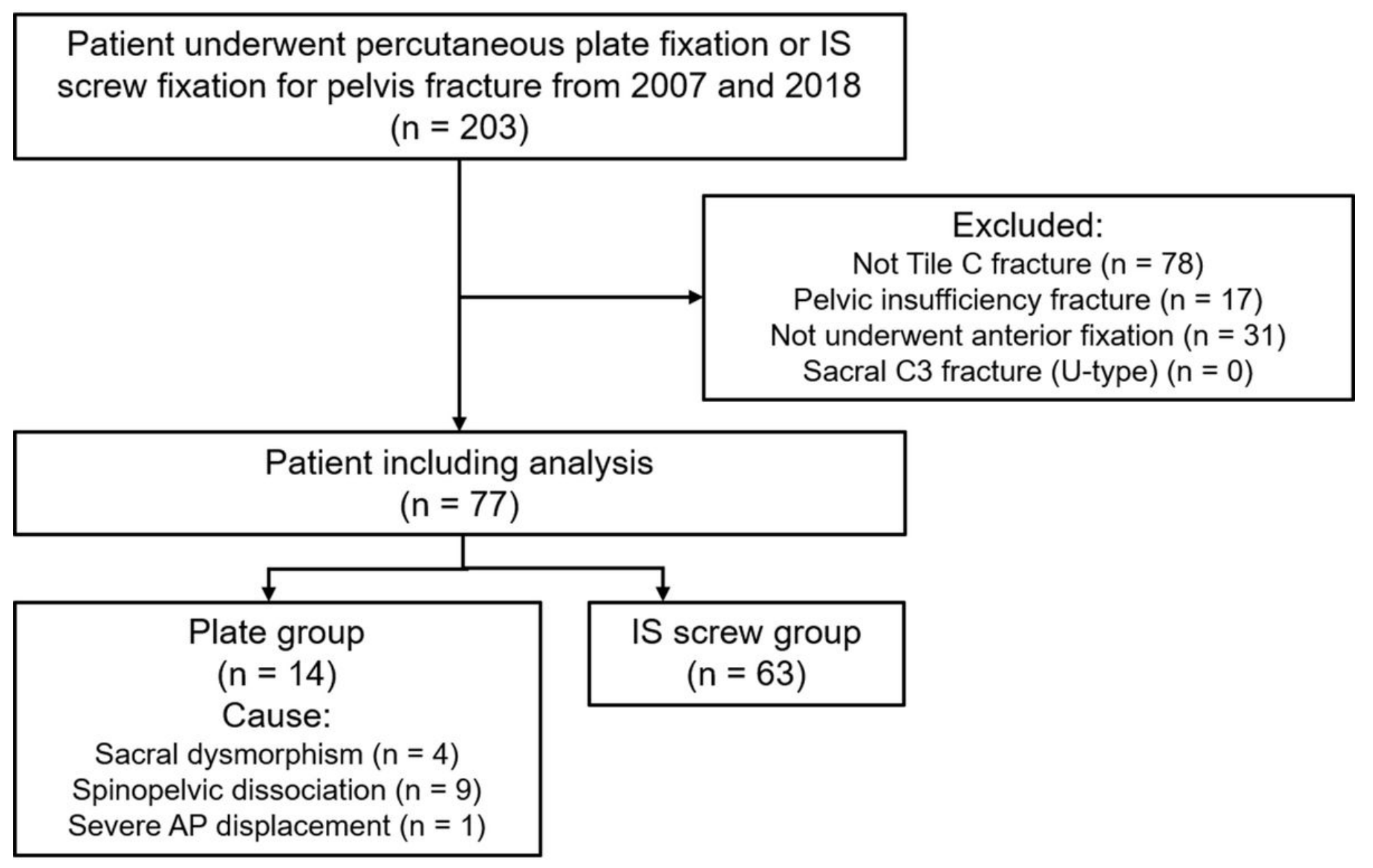

Figure 1

The flowchart of the patient exclusion process is based on the study criteria

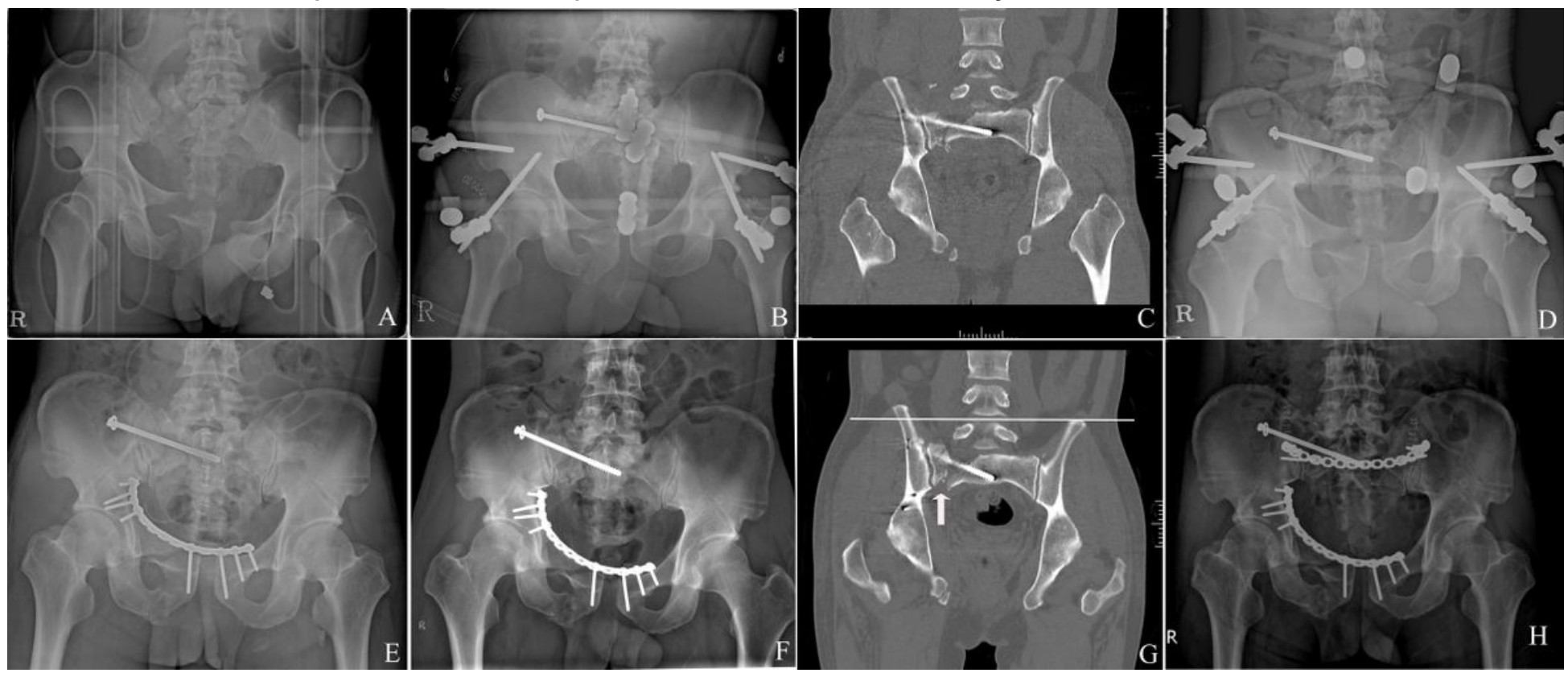

Figure 2 
(A) A 30-year-old man with a Tile C-type pelvic ring injury. (B) External fixation and iliosacral (IS) screw fixation on the day of injury. (C) Coronal reconstructed image showing a well-reduced posterior ring. (D) Post-injury radiograph at 4 weeks showing IS screw loosening. Definitive treatment was delayed due to unstable conditions of the patient with multiple trauma including aortic dissection. (E) Anterior plating 1month post-injury. (F) Pelvic radiograph showing IS screw migration at 8 weeks post-injury. (G) Coronal image at 8 weeks post-injury showing vertically displaced sacral alar (arrow) and upward migration of right pelvis (white line). $(\mathrm{H})$ Posterior transiliac plating was performed to acquire a more rigid fixation at 2 months post-injury.

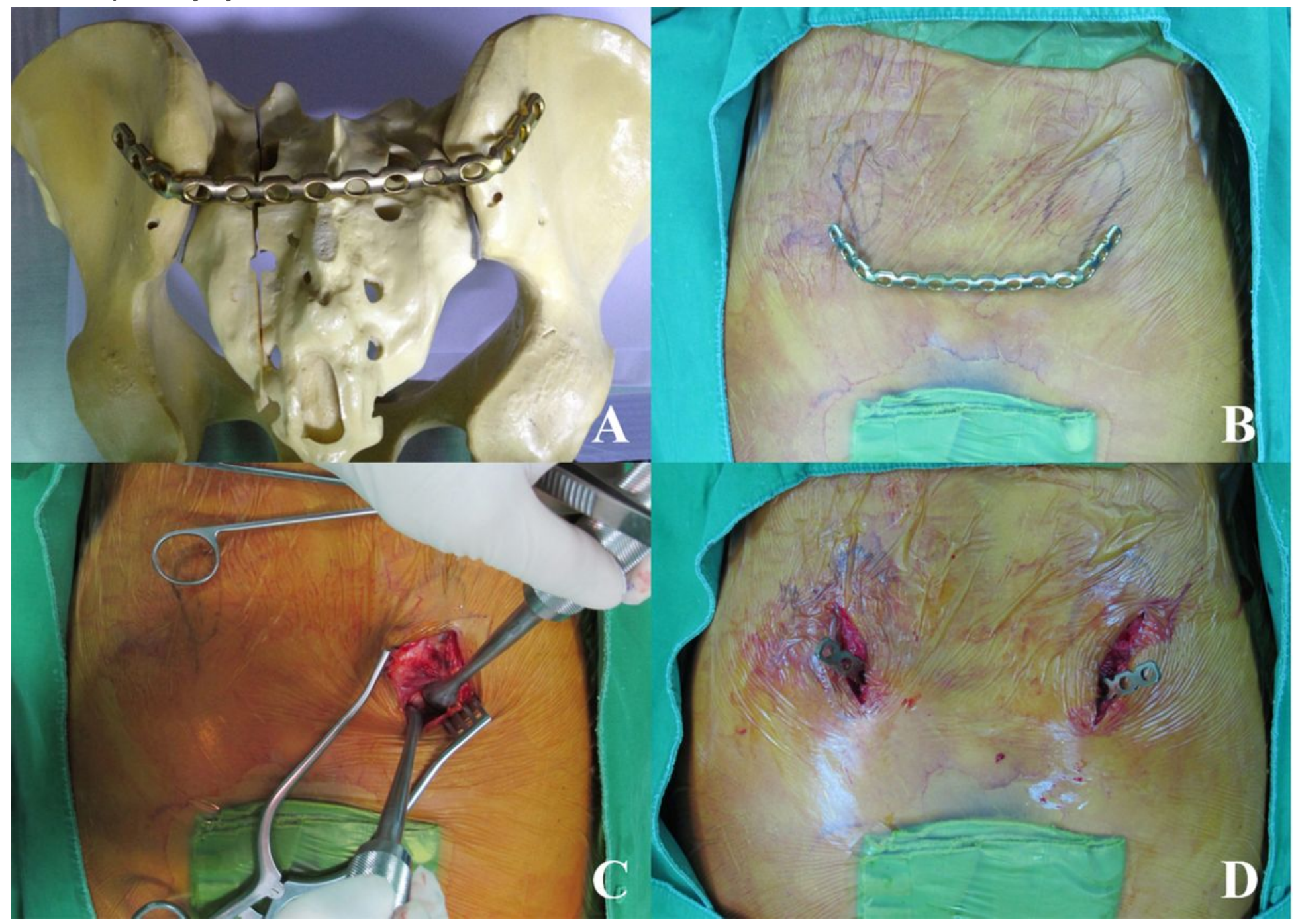

Figure 3

(A) A 30-year-old man with a Tile C-type pelvic ring injury. (B) External fixation and iliosacral (IS) screw fixation on the day of injury. (C) Coronal reconstructed image showing a well-reduced posterior ring. (D) Post-injury radiograph at 4 weeks showing IS screw loosening. Definitive treatment was delayed due to unstable conditions of the patient with multiple trauma including aortic dissection. (E) Anterior plating 1month post-injury. (F) Pelvic radiograph showing IS screw migration at 8 weeks post-injury. (G) Coronal image at 8 weeks post-injury showing vertically displaced sacral alar (arrow) and upward migration of 
right pelvis (white line). $(\mathrm{H})$ Posterior transiliac plating was performed to acquire a more rigid fixation at 2 months post-injury. 